\title{
SPLINE FITS: MODELLING THE OBSERVATIONS
}

\author{
I.L. ANDRONOV \\ Department of Astronomy, Odessa State University, \\ T.G.Shevchenko Park, Odessa 270014 Ukraine
}

The main results on the elaboration of the algorithms and programs for the search of the 'hidden periodicities' in the 'unevenly spaced' data, are discussed. They are mainly based on the application of the cubic spline-functions practically for all purposed of the variable star research - period determination, approximation of the phase curve, search for the possible period changes and/or the shape of the phase curve, detection of the periodic components in the case of the multi-harmonic oscillations, restoration of the smoothed function by removing the 'apparat function', numerical integration etc. Contrary to the commonly used splines with the arguments of the basic points coinciding with that of the real observations, we use the smoothing by the spline with the number of the basic points $\mathrm{m}$, which is smaller than the number of the observations $\mathrm{n}$. The main expressions for the corresponding fitting curves and their statistical properties were published by Andronov (1988, 1989), as well as the FORTRAN programs for the fits and the integration (Andronov, 1986, 1987, respectively).

For the evaluation of the smoothed curves at the small computers, the method of the 'Running parabolae' is proposed, the corresponding programs for which are significantly smaller as compared with that applying the cubic spline - functions (Andronov, 1990). The statistical properties of the periodograms obtained for the moments of the 'characteristic times' only are discussed by Andronov $(1988,1991)$.

\section{References}

Andronov I.L.: 1985, Preprint Ukr.Inst.Sci.-Tech.Res. (UkrNIINTI, Kiev) 131, 38 pp.

Andronov I.L.: 1986, Preprint UkrNIINTI 358, 64pp.

Andronov I.L.: 1987, Publ.Astr.Inst.Czechoslovakia 70, 161

Andronov I.L.: 1988, Astron. Nachr. 309, 121

Andronow I.L., 1989, Die Sterne 65, 20

Andronov I.L.: 1990, Cinem.\& Phys.Celest.Bodies (Kiev) 6, 87

Andronov I.L.: 1991, Cinem.\& Phys.Celest.Bodies (Kiev) 7, 78 